\title{
Safety and efficacy of the supracostal access for percutaneous nephrolithotomy: Our initial experience
}

\author{
Hossain M, Ullah ATMA, Regmi S, Rahman H, Kibria SAMG \\ Department of Urology, Bangabandhu Sheikh Mujib Medical University, Dhaka
}

\begin{abstract}
The aim of this study was to evaluate the safety and efficacy of the supracostal access for percutaneous nephrolithotomy (PCNL). Between July 2007 and June 2010, 122 patients underwent PCNL, of whom 28 (23\%) had supracostal access. All procedures were performed in a single sitting under general anesthesia. The data were analysed for indications, stone clearance rates and the complications associated with supracostal puncture. The indications for a supracostal access were staghorn stones $(50 \%)$, pelvis stones $(28.5 \%)$, calyceal stones in high-lying kidney (18\%) and upper ureter/ureteric stones (3.5\%). All tracts were made in the 11th intercostal space. Single tract access was used in 22 cases (78\%), but $6(22 \%)$ required a second tract. Additional punctures were required mainly for staghorn stones (4 out of 14). Overall, $82 \%$ of the patients were rendered stone free or had clinically insignificant residual stones with PCNL monotherapy, and this increased to $96 \%$ with secondary procedures. In patients with staghorn stones, they were completely cleared in $78 \%$. Overall complication rate was $28 \%$ and included hydrothorax in $3(10 \%)$ patients, which required insertion of a chest tube. One $(3.5 \%)$ patient developed haemothorax secondary to injury of the intercostal artery, pelvic perforation in $1(3.5 \%)$, perinephric collection in $1(3.5 \%)$, infection/sepsis in $2(7 \%)$. Except those patients who had complication, all other patient recovered uneventfully. Postoperative hospital stay ranged from 2 to 9 days. In conclusion, supracostal access gives high clearance rate with acceptable complications and should not be avoided for fear of chest complications.
\end{abstract}

\section{Introduction}

Percutaneous nephrolithotomy (PCNL) is the treatment of choice for staghorn stones, large renal stones and some upper ureteric stones 1. Successful removal requires the accurate placement of a percutaneous tract that provides direct access for stone manipulation. The optimal access for the staghorn, large upper calyceal and complex renal stone is through the upper pole posterior calyx, which at times is best accomplished by supracostal puncture ${ }^{2}$. However, supracostal puncture is a concern because of its potential pleural complications such as pneumothorax, hydrothorax and lung injury $^{3}$; hydrothorax being reported in 6-32\% of the procedures ${ }^{4,5}$.

In majority of renal calculi, access to the desired calyx is possible by a subcostal puncture. However, in most staghorn, upper ureteral and complex upper pole calculi, the subcostal approach fails to provide optimal access. In these cases, a supracostal approach provides direct access and thereby facilitates better stone clearance ${ }^{6}$. Usually, the upper pole of the kidney is more posterior and more medial than the lower pole, so access via a superior posterior calyx is more direct to the long axis of the kidney. This approach additionally facilitates the access to a lower calyx and the upper ureter. In complex inferior calyceal calculi, complete clearance may often not be possible through a single tract in an inferior calyx because of problems in negotiating the acute angles between calyces ${ }^{7}$. Supracostal puncture is also particularly useful in very obese patients and those with highlying kidneys. We evaluated the safety and efficacy of the supracostal access for the percutaneous removal of staghorn and complex renal stones.

\section{Materials and Methods}

Between July 2007 and June 2010, 122 patients underwent PCNL; supracostal access was obtained in 28 patients, comprising $23 \%$ of the procedures. The preoperative evaluation 
included hematocrit, renal function, urinalysis, and urine culture. Radiologic evaluation routinely included an intravenous urogram; ultrasonogram and split renal function measurement by DTPA were performed wherever indicated.

All the procedures were performed in a single stage under general anesthesia. After cystoscopic ureteral catheterization, the patient was positioned prone and a puncture was made above the lateral half of the 12 th rib lateral to the erector spinae, usually 7 to $8 \mathrm{cms}$ from the midline, under fluoroscopic guidance. All the supracostal punctures were made during full expiration to prevent parenchymal injury to the lung, and the needle advanced just above the upper border of the 12th rib to avoid damage to the intercostal vessels. Entry into the renal parenchyma was made in deep inspiration to provide full downward displacement of the kidney for easy access to the superior pole posterior calyx. Once the pelvicalyceal system was entered, the guidewire was manipulated down the ureter if possible or coiled in the distant calyx and subsequently the tract was dilated using serial fascial dilators and a 2830 F Amplatz sheath was placed. A standard 26 F nephroscope was then introduced through the Amplatz sheath and the stone was fragmented with the pneumatic lithotripter and removed. At the end of procedure, a 26-28 F chest tube was placed as nephrostomy tube. All patients had a chest X-ray and ultrasound scans soon after surgery to exclude pneumothorax or fluid collection. On suspicion of chest complication, a thoracic surgeon was consulted, and intercostal chest tube drainage was done if indicated.

Stone clearance was assessed with a plain abdominal X-ray at 1 or 2 days follow-up. Complete clearance was considered as no radiological evidence of residual stone disease. Asymptomatic residual, nonobstructing, nonstruvite stone fragments less than $5 \mathrm{~mm}$ in diameter were considered clinically insignificant residual fragments. Significant residual calculi were managed by ESWL. The data were analysed for the indications for supracostal access sites, clearance rates and postoperative complications.

\section{Results}

The 28 patients were in the age range of 2355 years (mean 39 years); 20(72\%) were males and $8(28 \%)$ were females. In $16(57 \%)$ patients the procedure was right-sided and left-sided in $12(43 \%)$. The majority (50\%) of them were partial and complete staghorn stones. Other indications were large pelvic stones $(28.5 \%)$, calyceal stones in high-lying kidneys (18\%) and upper ureteric stones (3.5\%) (Figure 1).

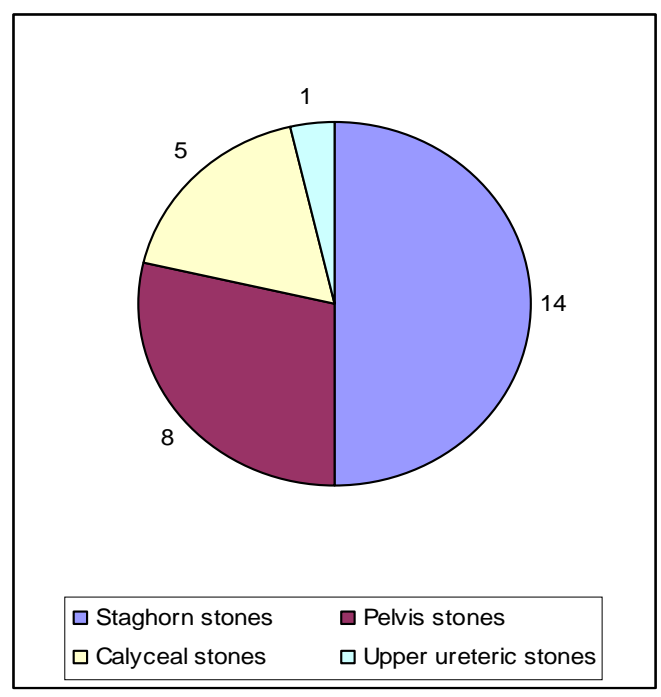

Figure 1: Stone distribution

Single-tract access was sufficient in $22(78 \%)$ cases; $6(22 \%)$ cases needed an additional access tract. Additional punctures were required mainly for staghorn stones (4 out of 14). The entry was through the superior calyx in $19(68 \%)$, middle calyx in $8(28.5 \%)$ and lower calyx in $1(3.5 \%)$ (Table I). In case of lower calyceal puncture, a higher relation of the lower calyx to the 12 th rib necessitated a supracostal puncture. This variation in calyceal entry is caused by a different anatomic distribution of calices above the 12th rib.

Table I: Site of puncture for access.

\begin{tabular}{lc}
\hline Site of puncture & Number $(\%)$ \\
\hline Supracostal access & $28(23)$ \\
Calyceal puncture & \\
Superior calyx & $19(68)$ \\
Middle calyx & $8(28.5)$ \\
Lower calyx & $1(3.5)$ \\
\hline
\end{tabular}

With PCNL monotherapy, stones were completely cleared in 23 cases (82\%). Significant residual fragments were present in 5 patients, of which one was lost to follow-up. The remaining 4 patients were later rendered stone free with multiple ESWL sessions (sandwich therapy). Thus, with later secondary procedures, the stone clearance rates improved 
to $96 \%$. There were $14(50 \%)$ patients with staghorn stones, which were completely cleared in $11(78 \%)$ patients, $3(22 \%)$ patients required ESWL for significant residual stone.

Table II: Complications of supracostal access.

\begin{tabular}{ll}
\hline Complications & Number $(\%)$ \\
\hline Hydrothorax & $3(10)$ \\
Intercostal vessel injury / Hemothorax & $1(3.5)$ \\
Pelvic perforation & $1(3.5)$ \\
Perinephric collection & $1(3.5)$ \\
Infection / Sepsis & $2(7)$ \\
Total & $8(28.5)$ \\
\hline
\end{tabular}

Complications developed in $8(28 \%)$ patients (Table II), with a chest complication in 4 (14\%). In 3 patients hydrothorax was detected on postoperative chest $\mathrm{X}$-ray which required insertion of a chest tube. A thoracic surgeon was consulted, and intercostal chest tube drainage was done. The mean duration of chest tube drainage was 4 days. The follow up X-ray was normal in all the patients. Haemothorax developed secondary to injury of the intercostal artery in 1 patient; this patient made an uneventful recovery after insertion of the chest tube and a blood transfusion. The chest tube was removed 6 days after surgery.

Pelvic perforation was noted in 1 patient during surgery but the procedure was continued, as there was no bleeding. However, this patient required nephrostomy drainage for longer duration (9 days, mean 5.5 days). The patient with perinephric collection was treated by conservative measures. Both the patients with sepsis recovered well with intravenous antibiotics and supportive measures. Blood transfusion was required in 3 patients. The mean postoperative hospital stay was 5.5 days (range 2-9 days).

\section{Discussion}

The outcome of PCNL is directly related to an optimal access tract. The majority of stones in the pelvis and mid or lower calyces can be easily reached via a subcostal puncture. However, for stones in the upper ureter, superior calyx and for staghorn stones, an approach through the superior calyx has clear advantages. Though technically more demanding, access through a superior calyx provides a straight tract along the axis of the kidney, with excellent visualization of upper and lower calyces, the pelvis, and the pelviureteric junction ${ }^{7}$. A straight tract also favours easier manipulation of the rigid scopes and forceps. This ability to operate along the long axis of the kidney causes less torque of the rigid nephroscope, thereby reducing the chances of excessive bleeding ${ }^{8}$. Invariably the superior calyceal approach is supracostal, which incurs few extra complications than subcostal access.

Staghorn stones are best approached through the supracostal puncture (Figure 2). A single puncture was used in $71.5 \%$ of the patients with staghorn stones, with additional punctures required in $28.5 \%$ to remove the middle calyceal extensions, which are difficult to see through the superior calyceal tract. Others have reported a $20-40 \%$ rate for additional punctures $^{9,10}$. The subcostal inferior calyx approach in staghorn stones has the problem of angulation and torque on the kidney, which may cause trauma and bleeding. Also it is particularly difficult to clear the residue in the superior calyx. Our analysis revealed good stone clearance rates $(78 \%)$ by PCNL monotherapy that are comparable with those noted by Kekre et al. $(79.5 \%)^{6}$ and Golijanin et al. $(87 \%)^{11}$.

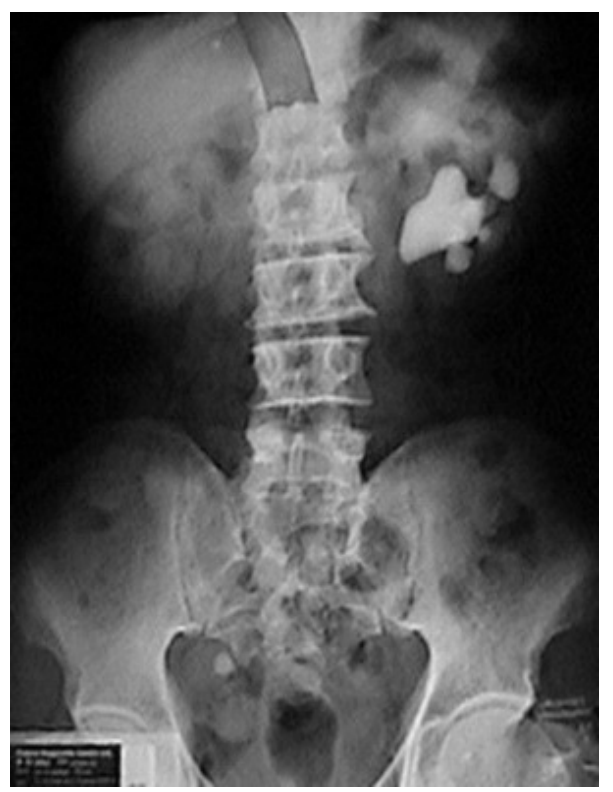

Figure 2: Staghorn stones are best approached via a supracostal puncture.

Supracostal approach is mandatory for superior calyceal stones as these stones are particularly difficult to approach through the inferior calyx because of the angulation of the tract. 
Upper ureteric stones are best approached via a supracostal tract (Figure 3). Although access through the middle or inferior calyx may be selected, it can lead to angulation between the working sheath and the pelvis, leading to difficulty in passing instruments or the lithoclast probe.

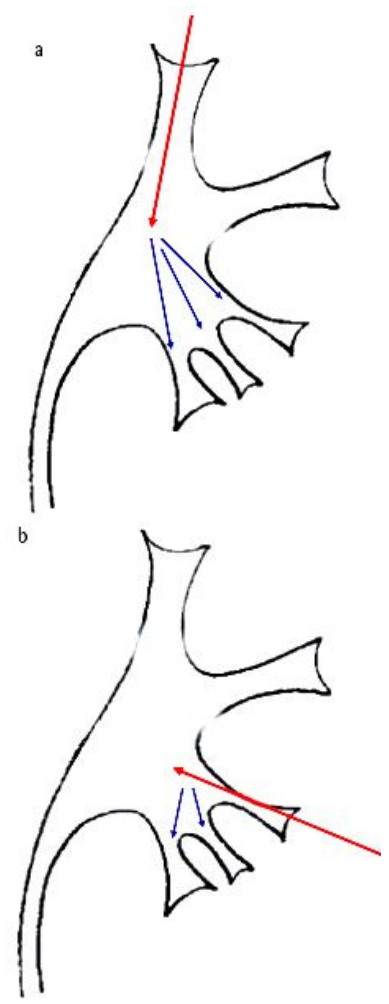

Figure 3: Line diagram showing the rationale for access to inferior polar calyces \& upper ureter (a); the difficult angles to be negotiated to access lower pole calyces if the puncture is made in one of these calyces (b).

The major disadvantage of supracostal access is the potential for pleural complications. Therefore a thorough knowledge of the anatomical relationships of the diaphragm, pleura and lung is important to avoid this risk. The parietal pleura is reflected to the level of 10 th rib in the mid-axillary line and posteriorly it is usually reflected obliquely at the midpoint of the 12th rib. In full expiration, the visceral pleura never descends to the level of the midpoint of the 12th rib. Hence, a supracostal puncture made in full expiration with optimal lateral approach will avoid the pleural space. The cause of hydrothorax has been attributed to accidental entry into the pleura and failure to seal the tract with the working sheath during the procedure or to inadequate drainage of the kidney afterward ${ }^{7}$. Overall, the occurrence of hydrothorax and hemothorax (13.5\%) in our series were comparable with those of earlier series $(1.7 \%-15 \%)^{6,11,12}$ and recovery was uneventful by conservative measures.

Another potential complication of supracostal access is the risk of injury to the lung, leading to tension pneumothorax. No such injury was encountered in the present series and was not reported by others. Injury to the viscera (liver or spleen) may occur with the more cephalad puncture, and thus we avoided access above the 11th rib. Haemothorax secondary to the laceration of the intercostal artery developed in one patient. Injury to the intercostal artery may be avoided by staying immediately above the upper border of the lower rib $^{7}$.

In conclusion, the supracostal superior calyceal approach provides optimum access to large upper calyceal and ureteric stones, being particularly suited to the percutaneous removal of complex staghorn stones. Although the morbidity is slightly higher than with a subcostal approach, this may be avoided to some extent by adhering to the basic principles of always puncturing in full expiration, sufficiently laterally to the margin of erector spinae muscle closer to the midscapular line, and always using a working sheath during nephroscopy and a well-draining nephrostomy tube after the procedure. Proper attention to the technique and intraoperative and postoperative monitoring can detect chest complications, and these can easily be managed with intercostal drainage without serious morbidity or death.

\section{References}

1. Galvin DJ, Pearle MS. The contemporary management of renal and ureteric calculi. BJU Int. 2006; 98:1283-1288.

2. Wolf JS Jr, Clayman RV. Percutaneous nephrolithotomy. What is its role in 1997. Urol Clin North Am. 1997; 24: 43-58.

3. Coleman CC, Kimura Y, Reddy P. Complications of nephrolithotomy. Semin Interv Radiol. 1984; 1: 70-74.

4. Narsmhan DL, Jacobsson B, Vijayan P. Percutaneous nephrolithotomy through intercostal approach. Acta Radiol. 1991; 32: 162-65.

5. Picus D, Weyman PJ, Clayman RV. Intercostal space nephrostomy for percutaneous stone removal. AJR. 1986; 147: 393-97.

6. Kekre NS, Gopalakrishnan GG, Gupta GG. Supracostal approach in percutaneous 
nephrolithotomy: experience with 102 cases. J Endourol. 2001; 15: 789-791.

7. Gupta R, Kumar A, Kapoor R. Prospective evaluation of safety and efficacy of the supracostal approach for percutaneous nephrolithotomy. BJU Int. 2002; 90: 809-813.

8. Munver R, Delvecchio FC, Newman GE. Critical analysis of supracostal access for percutaneous renal surgery. J Urol. 2001; 166: 1242-1246

9. Kertan RK, Kahn RK, Laberge JM. Percutaneous removal of renal staghorn calculi. AJR. 1985; 145: 797-801.
10. Adams GW, Oke EJ, Dunnikc NR. Percutaneous nephrolithotripsy of staghorn calculi. AJR 1985; 145: 803-7.

11. Golijanin D, Katz R, Verstanding A. The supracostal percutaneous nephrostomy for treatment of staghorn and complex kidney stones. J Endourol. 1998; 12: 403-405.

12. Lojanapiwat B, Prasopsuk S. Upper-pole access for percutaneous nephrolithotomy: comparison of supracostal and infracostal approaches. J Endourol. 2006; 20: 491-494. 